\title{
Greybody factors for a spherically symmetric Einstein-Gauss-Bonnet-de Sitter black hole
}

\author{
Cheng-Yong Zhang, ${ }^{1, *}$ Peng-Cheng $\mathrm{Li}^{2, \dagger}$ and Bin Chen ${ }^{1,2,3, *}$ \\ ${ }^{1}$ Center for High Energy Physics, Peking University, 5 Yiheyuan Road, Beijing 100871, China \\ ${ }^{2}$ Department of Physics and State Key Laboratory of Nuclear Physics and Technology, Peking University, \\ 5 Yiheyuan Road, Beijing 100871, China \\ ${ }^{3}$ Collaborative Innovation Center of Quantum Matter, 5 Yiheyuan Road, Beijing 100871, China
}

(Received 11 December 2017; published 7 February 2018)

\begin{abstract}
We study the greybody factors of the scalar fields in spherically symmetric Einstein-Gauss-Bonnet-de Sitter black holes in higher dimensions. We derive the greybody factors analytically for both minimally and nonminimally coupled scalar fields. Moreover, we discuss the dependence of the greybody factor on various parameters including the angular momentum number, the nonminimally coupling constant, the spacetime dimension, the cosmological constant, and the Gauss-Bonnet coefficient in detail. We find that the nonminimal coupling may suppress the greybody factor and the Gauss-Bonnet coupling could enhance it, but they both suppress the energy emission rate of Hawking radiation.
\end{abstract}

DOI: $10.1103 /$ PhysRevD.97.044013

\section{INTRODUCTION}

The black holes obey the laws of thermodynamics [1]. This inspires Hawking's pioneering works on the thermal radiation of the black hole, the so-called Hawking radiation $[2,3]$. Though in the vicinity of the black hole event horizon, the Hawking radiation is a blackbody radiation, determined by the temperature of the black hole, it becomes a greybody radiation at the asymptotic region since the radiation has to transverse an effective potential barrier. This effective potential barrier is highly sensitive to the structure of the black hole background. As a result, the Hawking radiation encodes important information about the black hole, including its mass, its charge, and its angular momentum. In general, the Hawking radiation of macroscopic black holes is too small to be detected. However, the Hawking radiation of the microscopic black holes could be detectable [4]. Especially the existence of extra spacelike dimensions [5-8] indicates that the tiny black hole may be created at the particle colliders [9-13] or in high energy cosmic-ray interactions [14-17]. Thus the associated Hawking radiation maybe observed at the $\mathrm{TeV}$ scale. As a result, significant number of works about the Hawking

\footnotetext{
*zhangcy0710@pku.edu.cn

†wlpch@pku.edu.cn

"bchen01@pku.edu.cn
}

Published by the American Physical Society under the terms of the Creative Commons Attribution 4.0 International license. Further distribution of this work must maintain attribution to the author(s) and the published article's title, journal citation, and DOI. Funded by SCOAP. radiation in higher dimensional spacetime have been done. For more extensive references one may consult the reviews [18-21].

In the asymptotic flat spacetimes, it has been found that the greybody factors for the waves of arbitrary spin and angular quantum number $l$ in any dimensions vanish in the zero-frequency limit [22-24], even for the nonminimally coupled scalar [25]. In the presence of a positive cosmological constant, the picture is different. The greybody factors of the Schwarzschild-de Sitter (SdS) black holes were studied both analytically and numerically in $d$ dimensions in [26], and it was found that the $l=0$ greybody factor was not vanishing even in the zerofrequency limit for a minimally coupled massless scalar (see also [27]). This implies that the cosmological constant has an important effect on the greybody factor as it leads to the fully delocalization of the zero-modes such that there is a finite probability for the zero-modes to transverse the region between the event horizon and the cosmological horizon [26]. The mass of the scalar or a nonminimally coupling constant breaks this relation, hence the greybody factors for arbitrary nonminimally coupled scalar partial modes in 4-dimensional spacetime tend to zero in the infrared limit [28].

In this paper, we consider the spherically symmetric dS black hole in the Einstein-Gauss-Bonnet (EGB) gravity. ${ }^{1}$ The EGB gravity is a special case of the Lovelock gravity which is the natural generalization of general relativity in higher dimensions [29]. As the most general metric theory

\footnotetext{
${ }^{1}$ In the following, we simply call such solution the EGB-dS black hole.
} 
of the gravity whose equations of motion are only the second order differential equations, the Lovelock gravity is ghost free and thus is especially attractive in the higherderivative gravity theories. Among the Lovelock gravity theories, the simplest one is the EGB gravity, which adds a fourth-derivative Gauss-Bonnet (GB) term to the EinsteinHilbert action

$$
\begin{aligned}
S_{G}= & \frac{1}{16 \pi G} \int d^{d} x \sqrt{-g}\left[R+\alpha\left(R_{\mu \nu \rho \sigma} R^{\mu \nu \rho \sigma}\right.\right. \\
& \left.\left.-4 R_{\mu \nu} R^{\mu \nu}+R^{2}\right)\right] .
\end{aligned}
$$

Here $\alpha$ is the Gauss-Bonnet coupling constant of dimension (length) ${ }^{2}$ and $R$ is the Ricci scalar. The Gauss-Bonnet coupling term appears in the low energy effective action of the heterotic string theory [30], where the coupling constant $\alpha$ is positive definite and inversely proportional to the string tension. Hence in this work we consider the case that $\alpha \geq 0$. $G$ is the $d$-dimensional Newton's constant. The GaussBonnet term is jut a topological term in $d=4$ spacetime and becomes nontrivial in $d>4$ spacetimes. It has been pointed out that if the Planck scale is of order $\mathrm{TeV}$, as suggested in some extra-dimension models, the coupling constant $\alpha$ could be measured by LHC through the detection of the spectrum of the Hawking radiation of the black hole [31]. Thus it is worth studying the greybody factor of the Hawking radiation of the GB black hole in higher dimensions, from both theoretical viewpoint and phenomenological purposes. For scalar and graviton emissions, the numerical studies of the GB black hole in an asymptotic flat spacetime were carried out in $[32,33]$. As mentioned in the last paragraph, the positive cosmological constant has significant effect on the greybody factor. In this paper we would like to compute the greybody factor of the Hawking radiation of the EGB-dS black hole analytically and discuss the effects of various parameters, especially the GB coupling constant, on the radiation.

The analytical study of the greybody factor in the SdS black hole has been well-developed. The analytical study in [26] was limited to the case of the lowest partial mode $(l=0)$ and the low energy part $(\omega \rightarrow 0)$ of the spectrum. A general expression for the greybody factor for arbitrary partial modes of a minimally or nonminimally coupled scalar in higher-dimensional SdS black hole was derived in [34]. The authors in [34] found an appropriate radial coordinate that allows them to integrate the field equations analytically and avoid the approximations on the metric tensor used in $[20,28]$. The comparison of the analytical result with the numerical result was done in [35]. For more studies, see [36-48]. Adopting a similar radial coordinate, we are able to derive the analytical results for the greybody factors for arbitrary partial modes of a scalar field in the EGB-dS black hole spacetime as well.

In Sec. II, we give the general background of the EGB$\mathrm{dS}$ black hole and the corresponding equation of motion for the scalar field. In Sec. III, we derive the analytical expression of the greybody factor using the matching method and discuss its low energy limit. We analyze the effects of various parameters on the greybody factor in Sec. IV and the energy emission of Hawking radiation in Sec. V. We end with the conclusion and discussion in Sec. VI.

\section{BACKGROUND}

The metric for a spherically symmetric Einstein-GaussBonnet-de Sitter black hole in $d$-dimensional spacetime is given by [30]

$$
\begin{aligned}
d s^{2} & =-h d t^{2}+\frac{d r^{2}}{h}+r^{2} d \Omega_{d-2}^{2}, \\
h & =1+\frac{r^{2}}{2 \tilde{\alpha}}\left(1-\sqrt{1+\frac{4 \tilde{\alpha} m}{r^{d-1}}+\frac{8 \tilde{\alpha} \Lambda}{(d-1)(d-2)}}\right) .
\end{aligned}
$$

The parameter $m$ is related to the mass of the black hole $M$ by $m=\frac{16 \pi G M}{(d-2) \Omega_{d-2}}$. In terms of the horizon radius $r_{h}, m$ can be expressed as

$$
m=r_{h}^{d-3}\left(1+\frac{\tilde{\alpha}}{r_{h}^{2}}-\frac{2 \Lambda r_{h}^{2}}{(d-1)(d-2)}\right) .
$$

Here $\tilde{\alpha}$ is related to the GB coupling constant $\alpha$ by $\tilde{\alpha}=\alpha(d-3)(d-4)$. In the limit $\tilde{\alpha} \rightarrow 0$, the metric returns to that of the SdS black hole. GB constant has significant effect on the stability of GB black holes. Through perturbation analysis, it was found that the EGB-dS black holes are unstable in certain parameter region. In our discussions, the parameters are restricted to the stable region given in [49-51] and will be chosen such that the spacetime always has two horizons, the black hole horizon $r_{h}$ and the cosmological horizon $r_{c}$.

We consider a general scalar field coupled to the gravity nonminimally

$$
S_{\Phi}=-\frac{1}{2} \int d^{d} x \sqrt{-g}\left[\xi \Phi^{2} R+\partial_{\mu} \Phi \partial^{\mu} \Phi\right] .
$$

Here $\xi$ is the nonminimally coupling constant with $\xi=0$ corresponding to the minimally coupled case. The equation of motion of the scalar field has the form

$$
\nabla_{\mu} \nabla^{\mu} \Phi=\xi R \Phi
$$

In a spherically symmetric background, we may make ansatz

$$
\Phi=e^{-i \omega t} \phi(r) Y_{(d-2)}^{l}(\Omega),
$$

where $Y_{(d-2)}^{l}(\Omega)$ are spherical harmonics on $S^{d-2}$. Then the angular part and the radial part are decoupled such that the radial equation becomes 
$\frac{1}{r^{d-2}} \frac{d}{d r}\left(h r^{d-2} \frac{d \phi}{d r}\right)+\left[\frac{\omega^{2}}{h}-\frac{l(l+d-3)}{r^{2}}-\xi R\right] \phi=0$.

Introducing $u(r)=r^{\frac{d-2}{2}} \phi(r)$, we get

$$
\frac{d^{2} u}{d r_{\star}^{2}}+\left(\omega^{2}-V\left(r_{\star}\right)\right) u=0,
$$

where $r_{\star}$ is the tortoise coordinate defined by $d r_{\star}=d r / h(r)$. The effective potential reads

$$
V\left(r_{\star}\right)=h\left[\frac{l(l+d-3)}{r^{2}}+\xi R+\frac{d-2}{2 r} h^{\prime}+\frac{(d-2)(d-4)}{4 r^{2}} h\right] .
$$

It is obvious that the effective potential vanishes at the two horizons. Its height increases with the angular momentum number $l$. Fixing the black hole horizon $r_{h}=1$, we can study the dependence of the profile of the effective potential on the angular momentum number $l$, the spacetime dimension $d$, the scalar coupling constant $\xi$, the cosmological constant $\Lambda$ and the GB coupling constant $\alpha$.

\section{GREYBODY FACTOR}

The radial equation (2.6) cannot be solved analytically over the whole space region. However, to read the greybody factor, it is not necessary to solve the equation exactly. Instead, one can solve the equation in two regions separately, namely near the black hole horizon and the cosmological horizon regions, and then paste the solutions in the intermediate region. In this procedure, the effect of the cosmological constant should be put under control in order to make the result as accurate as possible [34].

\section{A. Near the event horizon}

In the near event horizon region $r \sim r_{h}$, similar to the case of SdS, we perform the following transformation

$$
\begin{aligned}
r \rightarrow f(r) & =\frac{h}{1-\tilde{\Lambda} r^{2}}, \\
\tilde{\Lambda} & =-\frac{1}{2 \tilde{\alpha}}\left(1-\sqrt{1+\frac{8 \tilde{\alpha} \Lambda}{(d-1)(d-2)}}\right) .
\end{aligned}
$$

The new variable $f$ ranges from 0 to 1 as $r$ runs from $r_{h}$ to the region $r \gg r_{h}$. Its derivative satisfies

$$
\frac{d f}{d r}=\frac{1-f}{r} \frac{A(r)}{1-\tilde{\Lambda} r^{2}}
$$

with
$A(r)=-2+\frac{d-1}{2}\left(1+\frac{1}{\sqrt{1+\frac{4 \tilde{\alpha} m}{(1+2 \tilde{\alpha} \tilde{\Lambda})^{2}} \frac{1}{r^{d-1}}}}\right)\left(1-\tilde{\Lambda} r^{2}\right)$

in which the mass can be expressed as

$$
m=r_{h}^{d-3}\left(1-\tilde{\Lambda} r_{h}^{2}\right)\left[1+\frac{\tilde{\alpha}\left(1+\tilde{\Lambda} r_{h}^{2}\right)}{r_{h}^{2}}\right]
$$

When $\tilde{\alpha} \rightarrow 0$, it returns to the case of the SdS black hole, namely $A_{S d S}=-2+(d-1)\left(1-\tilde{\Lambda} r^{2}\right)$.

Using the new variable, the radial equation near the even horizon becomes

$$
\begin{aligned}
f(1 & -f) \frac{d^{2} \phi}{d f^{2}}+\left(1-B_{h} f\right) \frac{d \phi}{d f} \\
& +\left[-\frac{\left(\omega r_{h}\right)^{2}}{A_{h}^{2}}+\frac{\left(\omega r_{h}\right)^{2}}{A_{h}^{2} f}-\frac{\lambda_{h}\left(1-\tilde{\Lambda} r_{h}^{2}\right)}{A_{h}^{2}(1-f)}\right] \phi=0 .
\end{aligned}
$$

in which

$B_{h}=2-\frac{1-\tilde{\Lambda} r_{h}^{2}}{A_{h}^{2}}\left[(d-3) A_{h}+r A^{\prime}\left(r_{h}\right)\right]$,

$\lambda_{h}=l(l+d-3)+\xi R^{(h)} r_{h}^{2}$,

where $A_{h}=A\left(r_{h}\right)$ and $R^{(h)}=-h^{\prime \prime}+\left.(d-2) \frac{-2 r h^{\prime}+(d-3)(1-h)}{r^{2}}\right|_{r_{h}}$ is the Ricci scalar on the event horizon. In the derivation of this equation we have used the approximation

$$
\frac{\left(\omega r_{h}\right)^{2}}{A_{h}^{2} f(1-f)} \sim \frac{\left(\omega r_{h}\right)^{2}(1-f)}{A_{h}^{2} f}=-\frac{\left(\omega r_{h}\right)^{2}}{A_{h}^{2}}+\frac{\left(\omega r_{h}\right)^{2}}{A_{h}^{2} f},
$$

near the event horizon $f \sim 0$. The reason is that the solution of the original radial equation has cusps due to the poles of Gamma function, the unphysical behavior can be avoided by using this approximation. ${ }^{2}$

This is in fact a Fuchsian equation with three singularities $f=0,1, \infty$. To be clearer, make a redefinition $\phi=f^{\alpha_{1}}(1-f)^{\beta_{1}} W(f)$, Eq. (3.5) becomes

$$
\begin{gathered}
f(1-f) \frac{d^{2} W}{d f^{2}}+\left[1+2 \alpha_{1}-\left(2 \alpha_{1}+2 \beta_{1}+B_{h}\right) f\right] \frac{d W}{d f} \\
-\frac{\omega^{2} r_{h}^{2}+A_{h}^{2}\left(\alpha_{1}+\beta_{1}\right)\left(B_{h}+\alpha_{1}+\beta_{1}-1\right)}{A_{h}^{2}} W=0 .
\end{gathered}
$$

in which the coefficients are given by

\footnotetext{
${ }^{2}$ We thank Pappas and Kanti for their correspondences on this point.
} 
$\alpha_{1}= \pm i \frac{\omega r_{h}}{A_{h}}$,

$\beta_{1}=\frac{1}{2}\left(2-B_{h} \pm \sqrt{\left(2-B_{h}\right)^{2}+\frac{4 \lambda_{h}\left(1-\tilde{\Lambda} r_{h}^{2}\right)}{A_{h}^{2}}}\right)$.

The solution of the differential equation (3.8) is the standard hypergeometric function $F\left(a_{1}, b_{1}, c_{1}, f\right)$ with parameters $a_{1}, b_{1}, c_{1}$ being

$a_{1}=\alpha_{1}+\beta_{1}+\frac{1}{2}\left(B_{h}-1+\sqrt{\left(1-B_{h}\right)^{2}-\frac{4 \omega^{2} r_{h}^{2}}{A_{h}^{2}}}\right)$,

$b_{1}=\alpha_{1}+\beta_{1}+\frac{1}{2}\left(B_{h}-1-\sqrt{\left(1-B_{h}\right)^{2}-\frac{4 \omega^{2} r_{h}^{2}}{A_{h}^{2}}}\right)$,

$c_{1}=1+2 \alpha_{1}$.

Considering the relation between $\phi(f)$ and $W(f)$, near the event horizon the radial function $\phi(f)$ has the following form

$$
\begin{aligned}
\phi_{H}= & A_{1} f^{\alpha_{1}}(1-f)^{\beta_{1}} F\left(a_{1}, b_{1}, c_{1}, f\right) \\
& +A_{2} f^{-\alpha_{1}}(1-f)^{\beta_{1}} F\left(1+a_{1}-c_{1}, 1+b_{1}-c_{1}, 2-c_{1}, f\right) .
\end{aligned}
$$

where $A_{1,2}$ are the constant coefficients. Near the event horizon,

$$
\phi_{H} \simeq A_{1} f^{\alpha_{1}}+A_{2} f^{-\alpha_{1}}, \quad \text { and } f \propto e^{A_{h} r_{\star} / r_{h}} .
$$

Imposing the ingoing boundary condition near the event horizon and choosing $\alpha_{1}=-i \frac{\omega r_{h}}{A_{h}}$, we should set $A_{2}=0$. Furthermore, the convergence of the hypergeometric function requires the real part $\operatorname{Re}\left(c_{1}-a_{1}-b_{1}\right)>0$. Thus we have to take the "-" branch of $\beta_{1}$. In the end, the solution near the event horizon is of the form

$$
\phi_{H}=A_{1} f^{\alpha_{1}}(1-f)^{\beta_{1}} F\left(a_{1}, b_{1}, c_{1}, f\right) .
$$

\section{B. Near the cosmological horizon}

The solution in the near cosmological horizon region can be solved similarly. The function $h$ in the metric can be approximated by $[20,28,34]$

$$
h(r)=1-\tilde{\Lambda} r^{2}-\left(\frac{r_{h}}{r}\right)^{d-3}\left(1-\tilde{\Lambda} r_{h}^{2}\right) \sim \tilde{h}=1-\tilde{\Lambda} r^{2} .
$$

$\tilde{h}$ ranges from 0 , at $r=r_{c}$, to 1 as $r \ll r_{c}$. In the above approximation, the larger $r_{c}$ or the smaller $\tilde{\Lambda}$ leads to more accurate results. The approximation also becomes more accurate for a larger spacetime dimension $d$.
Making the change of variable $r \rightarrow \tilde{h}(r)$, near the cosmological horizon, we have

$$
\begin{aligned}
\tilde{h} & (1-\tilde{h}) \frac{d^{2} \phi}{d \tilde{h}^{2}}+\left(1-\frac{d+1}{2} \tilde{h}\right) \frac{d \phi}{d \tilde{h}} \\
+ & {\left[\frac{\left(\omega r_{c}\right)^{2}}{4 \tilde{h}}-\frac{l(l+d-3)}{4(1-\tilde{h})}-\frac{\xi R^{(c)} r_{c}^{2}}{4}\right] \phi=0, }
\end{aligned}
$$

where $R^{(c)}=-\tilde{h}^{\prime \prime}+\left.(d-2) \frac{-2 r \tilde{h}^{\prime}+(d-3)(1-\tilde{h})}{r^{2}}\right|_{r_{c}}$ is the Ricci scalar at $r_{c}$. After a replacement $\phi(\tilde{h})=\tilde{h}^{\alpha_{2}}(1-\tilde{h})^{\beta_{2}} X(\tilde{h})$, we get

$$
\begin{gathered}
(1-\tilde{h}) \tilde{h} \frac{d^{2} X}{d \tilde{h}^{2}}+\left[1+2 \alpha_{2}-\left(2 \alpha_{2}+2 \beta_{2}+\frac{d+1}{2}\right) \tilde{h}\right] \frac{d X}{d \tilde{h}} \\
-\frac{2\left(\alpha_{2}+\beta_{2}\right)\left(\alpha_{2}+\beta_{2}+d-1\right)+\xi R^{(c)} r_{c}^{2}}{4} X=0,
\end{gathered}
$$

in which

$$
\alpha_{2}= \pm i \frac{\omega r_{c}}{2}, \quad \beta_{2}=-\frac{d+l-3}{2} \quad \text { or } \quad \frac{l}{2} .
$$

The solution of the differential equation (3.14) could be written in terms of the hypergeometric functions as well. Therefore, around the cosmological horizon, the radial equation can be solved by

$$
\begin{aligned}
\phi_{C}= & B_{1} \tilde{h}^{\alpha_{2}}(1-\tilde{h})^{\beta_{2}} F\left(a_{2}, b_{2}, c_{2}, \tilde{h}\right) \\
& +B_{2} \tilde{h}^{-\alpha_{2}}(1-\tilde{h})^{\beta_{2}} F\left(1+a_{2}-c_{2}, 1+b_{2}-c_{2}, 2-c_{2}, \tilde{h}\right),
\end{aligned}
$$

with the parameters

$$
\begin{aligned}
& a_{2}=\alpha_{2}+\beta_{2}+\frac{d-1+\sqrt{(d-1)^{2}-4 \xi R^{(c)} r_{c}^{2}}}{4}, \\
& b_{2}=\alpha_{2}+\beta_{2}+\frac{d-1-\sqrt{(d-1)^{2}-4 \xi R^{(c)} r_{c}^{2}}}{4}, \\
& c_{2}=1+2 \alpha_{2} .
\end{aligned}
$$

Here $B_{1,2}$ are constant coefficients. The convergence of the hypergeometric function requires $\operatorname{Re}\left(c_{2}-a_{2}-b_{2}\right)>0$ such that we have to take $\beta_{2}=-\frac{d+l-3}{2}$.

Since the effective potential vanishes at $r_{c}$, the solution is expected to be comprised of the plane waves. Indeed, we have

$$
\phi_{C}=B_{1} e^{-i \omega r_{\star}}+B_{2} e^{i \omega r_{\star}}
$$

where $r_{\star}=\frac{1}{2} r_{c} \ln \frac{r / r_{c}+1}{r / r_{c}-1}$ is the tortoise coordinate near $r_{c}$. The first and second parts correspond to the ingoing and outgoing waves, respectively. The sign in $\alpha_{2}$ just 
interchanges the ingoing and outgoing waves. We take $\alpha_{2}=$ $i \frac{\omega r_{c}}{2}$ here. In contrast to what happens at the black hole horizon, both the ingoing and outgoing waves are now allowed. It is in fact their amplitudes that define the greybody factor for the emission of the scalar fields by the back hole. The greybody factor is given by

$$
\left|\gamma_{\omega l}\right|^{2}=1-\left|\frac{B_{2}}{B_{1}}\right|^{2}
$$

\section{Matching the solutions in the intermediate region}

Now we have the asymptotic solutions in the near event horizon region and the near cosmological horizon region. In order to complete the solution, we must ensure that the two asymptotic solutions, $\phi_{H}$ and $\phi_{C}$ can be smoothly pasted at the intermediate region.

\section{Black hole horizon}

First let us consider the near black hole horizon solution. Due to the fact that in the intermediate region $r \gg r_{h}$, the variable $f \rightarrow 1$, we can use the following relation for the hypergeometric function

$$
\begin{aligned}
F(a, b, c ; f)= & \frac{\Gamma(c) \Gamma(c-a-b)}{\Gamma(c-a) \Gamma(c-b)} \\
& \times F(a, b, a+b-c+1 ; 1-f) \\
& +(1-f)^{c-a-b} \frac{\Gamma(c) \Gamma(a+b-c)}{\Gamma(a) \Gamma(b)} \\
& \times F(c-a, c-b, c-a-b+1 ; 1-f)
\end{aligned}
$$

to shift the argument from $f$ to $1-f$. For simplicity we consider the case $\Lambda r_{h}^{2} \ll 1$. Then in the region where $r \gg r_{h}$, we have $A_{h} \simeq d-3$. This is reasonable only if $\Lambda r^{2} \simeq r^{2} / r_{c}^{2} \ll 1$. For $r \gg r_{h}$, from (3.1) we have

$$
h \rightarrow 1-\tilde{\Lambda} r^{2}+\mathcal{O}\left(\frac{r_{h}^{d-3}}{r^{d-3}}\right) .
$$

Then the Ricci scalar $R^{(h)} \rightarrow \frac{2 d \Lambda}{d-2}$. Thus if $\xi$ is not too big, the term $\xi R^{(h)} r_{h}^{2} \rightarrow \xi \frac{2 d \Lambda r_{h}^{2}}{d-2} \ll 1$ and can be omitted. Therefore, we have $B_{h} \simeq 1, \beta_{1} \simeq-\frac{l}{d-3}$.

Now we have

$$
\begin{aligned}
1-f & \simeq\left(1+\frac{\tilde{\alpha}}{r_{h}^{2}}\right)\left(\frac{r_{h}}{r}\right)^{d-3} \\
\beta_{1}+c_{1}-a_{1}-b_{1} & \simeq \frac{l+d-3}{d-3} .
\end{aligned}
$$

In the intermediate region $r \gg r_{h}$, the solution (3.12) can be expanded into the form

$$
\phi_{H} \simeq \Sigma_{2} r^{l}+\Sigma_{1} r^{-l-d+3}
$$

where

$$
\begin{aligned}
& \Sigma_{1}=A_{1} \frac{\Gamma\left(c_{1}\right) \Gamma\left(a_{1}+b_{1}-c_{1}\right)}{\Gamma\left(a_{1}\right) \Gamma\left(b_{1}\right)}\left(1+\frac{\tilde{\alpha}}{r_{h}^{2}}\right)^{\frac{l+d-3}{d-3}} r_{h}^{l+d-3}, \\
& \Sigma_{2}=A_{1} \frac{\Gamma\left(c_{1}\right) \Gamma\left(c_{1}-a_{1}-b_{1}\right)}{\Gamma\left(c_{1}-a_{1}\right) \Gamma\left(c_{1}-b_{1}\right)}\left(1+\frac{\tilde{\alpha}}{r_{h}^{2}}\right)^{\frac{-l}{d-3}} r_{h}^{-l} .
\end{aligned}
$$

Note that the aforementioned approximations are applicable only for the expressions involving the factor $(1-f)$ and not for the parameters in the Gamma function to increase the validity of the analytical results [34].

\section{Cosmological horizon}

Now let us turn to the solution near the cosmological horizon. Similar to the treatment above, we may shift the argument of the hypergeometric function from $\tilde{h}$ to $1-\tilde{h}$ since for the intermediate region $\tilde{h} \rightarrow 1$. We still work with a small cosmological constant. In the region where $r \ll r_{c}$, we have

$$
1-\tilde{h} \simeq\left(\frac{r}{r_{c}}\right)^{2}
$$

and $\beta_{2} \simeq-\frac{l+d-3}{2}, \beta_{2}+c_{2}-a_{2}-b_{2} \simeq l / 2$. Following the similar procedure, we get

$\phi_{C} \simeq\left(\Sigma_{3} B_{1}+\Sigma_{4} B_{2}\right) r^{-(l+d-3)}+\left(\Sigma_{5} B_{1}+\Sigma_{6} B_{2}\right) r^{l}$

where

$$
\begin{aligned}
& \Sigma_{3}=\frac{\Gamma\left(c_{2}\right) \Gamma\left(c_{2}-a_{2}-b_{2}\right)}{\Gamma\left(c_{2}-a_{2}\right) \Gamma\left(c_{2}-b_{2}\right)} r_{c}^{l+d-3}, \\
& \Sigma_{4}=\frac{\Gamma\left(2-c_{2}\right) \Gamma\left(c_{2}-a_{2}-b_{2}\right)}{\Gamma\left(1-a_{2}\right) \Gamma\left(1-b_{2}\right)} r_{c}^{l+d-3}, \\
& \Sigma_{5}=\frac{\Gamma\left(c_{2}\right) \Gamma\left(a_{2}+b_{2}-c_{2}\right)}{\Gamma\left(a_{2}\right) \Gamma\left(b_{2}\right)} r_{c}^{-l}, \\
& \Sigma_{6}=\frac{\Gamma\left(2-c_{2}\right) \Gamma\left(a_{2}+b_{2}-c_{2}\right)}{\Gamma\left(a_{2}-c_{2}+1\right) \Gamma\left(b_{2}-c_{2}+1\right)} r_{c}^{-l} .
\end{aligned}
$$

It is obvious that solutions (3.24) and (3.27) have the same power-law. Identifying the coefficients of the same powers of $r$ in (3.24) and (3.27), we get the relations

$$
\Sigma_{3} B_{1}+\Sigma_{4} B_{2}=\Sigma_{1}, \quad \Sigma_{5} B_{1}+\Sigma_{6} B_{2}=\Sigma_{2} .
$$

Solving the constraints and plugging them into the expression for the greybody factor for the emission of scalar fields by a higher dimensional EGB-dS black hole, we get

$$
\left|\gamma_{\omega l}\right|^{2}=1-\left|\frac{\Sigma_{2} \Sigma_{3}-\Sigma_{1} \Sigma_{5}}{\Sigma_{1} \Sigma_{6}-\Sigma_{2} \Sigma_{4}}\right|^{2} .
$$

This expression takes the same form as that for the Einstein gravity [34]. But due to the differences among the explicit 
expressions of $\Sigma$ s, it depends not only on the cosmological constant $\Lambda$ and the non-minimal coupling $\xi$, but also on the GB coupling constant $\alpha$.

As mentioned in [34], the greybody factor (3.30) is more accurate for a smaller cosmological constant and a larger distance between $r_{h}$ and $r_{c}$. On the other hand, in contrast with all the previous similar matching procedures here we do not make any assumption on the energy $\omega$ in the approximation, thus it might be possible that our analytical result can be valid beyond the low energy region. However, as we will see in the following section there are obvious deviations in the high energy region from the reasonable expected results, which means that the matching procedure only applies to the low energy region. This is because that the continuations of the asymptotic solutions near the event/cosmological horizon deviate from the exact solution in the intermediate region so the higher energy modes lead to larger deviations. Instead, one can numerically integrate the radial equation (2.6) in the intermediate region to get the more exact greybody factors for high energy modes. We leave this to future work.

\section{Low energy limit}

As we mentioned above, the analytical result of the greybody factor is only valid for low energy modes, therefor before analyzing the effects of various parameters on the greybody factor, we derive the low energy limit of the greybody factor in this subsection.

\section{Minimal coupling $\xi=0$ and dominant mode $l=0$}

Let us consider the minimally coupling $\xi=0$ case and the dominant mode $l=0$ first. In this case, we obtain

$$
\begin{aligned}
& \Sigma_{1} \sim A_{1} \frac{i \omega}{2-B_{h 0}}\left(1+\frac{\tilde{\alpha}}{r_{h}^{2}}\right) \frac{1}{A_{h 0}} r_{h}^{d-2}+O\left(\omega^{2}\right), \\
& \Sigma_{2} \sim A_{1}+O(\omega), \quad \Sigma_{3} \sim \frac{i \omega}{d-3} r_{c}^{d-2}+O\left(\omega^{2}\right), \\
& \Sigma_{4} \sim \frac{-i \omega}{d-3} r_{c}^{d-2}+O\left(\omega^{2}\right), \quad \Sigma_{5,6} \sim 1+O(\omega)
\end{aligned}
$$

where

$$
\begin{aligned}
A_{h 0}= & \frac{(d-3) r_{h}^{2}+(d-5) \tilde{\alpha}}{r_{h}^{2}+2 \tilde{\alpha}}, \\
B_{h 0}= & \frac{(d-3) r_{h}^{2}-4 \tilde{\alpha}}{(d-3) r_{h}^{2}+(d-5) \tilde{\alpha}}, \\
\lambda_{h 0}= & l(l+d-3)+(d-1) \tilde{\alpha} \xi \\
& \times \frac{(2-d) r_{h}^{4}+4 r_{h}^{2} \tilde{\alpha}+2(d+1) \tilde{\alpha}^{2}}{\left(r_{h}^{2}+2 \tilde{\alpha}\right)^{3}} .
\end{aligned}
$$

Then the greybody factor becomes

$\left|\gamma_{\omega l}\right|^{2}=\frac{4(d-3) A_{h 0}\left(2-B_{h 0}\right)\left(1+\frac{\tilde{\alpha}}{r_{h}^{2}}\right)\left(r_{h} r_{c}\right)^{d-2}}{\left[(d-3)\left(1+\frac{\tilde{\alpha}}{r_{h}^{2}}\right) r_{h}^{d-2}+A_{h 0}\left(2-B_{h 0}\right) r_{c}^{d-2}\right]^{2}}+O(\omega)$

Thus the scalar particle with very low energy has a nonvanishing probability of being emitted by a higher dimensional EGB-dS black hole. This is in fact a characteristic feature of the propagation of free massless scalar in the $\mathrm{dS}$ spacetime. However, the GB term changes the value of the greybody factor. For instance, for a small $\tilde{\alpha}$, up to the first order of $\tilde{\alpha}$,

$$
\begin{aligned}
\left|\gamma_{\omega l}\right|^{2}= & \frac{4\left(r_{h} r_{c}\right)^{d-2}}{\left(r_{h}^{d-2}+r_{c}^{d-2}\right)^{2}}+\frac{4 r_{c}^{d-2} r_{h}^{d-2}\left(r_{c}^{d-2}-r_{h}^{d-2}\right)}{\left(r_{c}^{d-2}+r_{h}^{d-2}\right)^{3}} \frac{\tilde{\alpha}}{r_{h}^{2}} \\
& +O\left(\omega, \tilde{\alpha}^{2}\right) .
\end{aligned}
$$

We see that $\tilde{\alpha}$ increases the greybody factor of massless scalar in the EGB-dS black hole background. When $\tilde{\alpha} \rightarrow 0$, we reproduce the low energy greybody factor for the mode $l=0$, in accordance to the previous higher dimensional analysis $[20,26,34]$.

\section{Nonminimally coupling case $\xi \neq 0$}

Now we calculate the low energy greybody factor for a nonminimally coupled scalar. In this case, we can expand the combinations in the low energy limit as

$$
\begin{aligned}
& \Sigma_{2} \Sigma_{3}=E+i \Sigma_{231} \omega+\Sigma_{232} \omega^{2}, \\
& \Sigma_{1} \Sigma_{5}=K+i \Sigma_{151} \omega+\Sigma_{152} \omega^{2}, \\
& \Sigma_{2} \Sigma_{4}=E+i \Sigma_{241} \omega+\Sigma_{242} \omega^{2}, \\
& \Sigma_{1} \Sigma_{6}=K+i \Sigma_{161} \omega+\Sigma_{162} \omega^{2} .
\end{aligned}
$$

in which $E, K, \Sigma_{231}, \Sigma_{232}, \Sigma_{151}, \Sigma_{152}, \Sigma_{241}, \Sigma_{242}, \Sigma_{161}, \Sigma_{162}$ are the expansion coefficients, whose explicit expressions are lengthy and will not be given here. The final result for the greybody factor turns out to be

$$
\left|\gamma_{\omega l}\right|^{2}=\frac{4 \pi^{8}\left(r_{c} r_{h}\right)^{d+2 l+3} R_{C} R_{H}\left(1+\frac{\tilde{\alpha}}{r_{h}^{2}}\right)^{\frac{2 l+d+3}{d-3}}}{\sin ^{2}(\pi \delta) \sin ^{2}(\pi \epsilon) \sin ^{2}\left(\pi \eta_{+}\right) \sin ^{2}\left(\pi \eta_{-}\right)(\delta+\epsilon-1)\left(\eta_{+}+\eta_{-}-1\right) C^{2}} \omega^{2}+O\left(\omega^{3}\right),
$$


in which $R_{H}=\frac{r_{h}}{A_{h 0}}, R_{C}=\frac{r_{c}}{2}$ and

$$
\begin{aligned}
\delta & =\frac{1}{2}\left(B_{h 0}-\sqrt{\left.\left(2-B_{h 0}\right)^{2}+\frac{4 \lambda_{h 0}}{A_{h 0}^{2}}\right)},\right. \\
\epsilon & =\frac{1}{2}\left(2-B_{h 0}-\sqrt{\left(2-B_{h 0}\right)^{2}+\frac{4 \lambda_{h 0}}{A_{h 0}^{2}}}\right), \\
\eta_{ \pm} & =\frac{5-d-2 l \pm \sqrt{(d-1)^{2}-4 \xi R^{(c)} r_{c}^{2}}}{4},
\end{aligned}
$$

and

$$
\begin{aligned}
C= & r_{c}^{3} r_{h}^{d+2 l}\left(1+\frac{\tilde{\alpha}}{r_{h}^{2}}\right)^{\frac{d+2 l}{d-3}} \Gamma(1-\delta) \Gamma(1-\epsilon) \Gamma(\delta+\epsilon-1) \\
& \times \Gamma\left(1-\eta_{+}\right) \Gamma\left(1-\eta_{-}\right) \Gamma\left(\eta_{+}+\eta_{-}-1\right) \\
& -r_{c}^{d+2 l} r_{h}^{3}\left(1+\frac{\tilde{\alpha}}{r_{h}^{2}}\right)^{\frac{3}{d-3}} \Gamma(\delta) \Gamma(\epsilon) \Gamma(1-\delta-\epsilon) \\
& \times \Gamma\left(\eta_{+}\right) \Gamma\left(\eta_{-}\right) \Gamma\left(1-\eta_{+}-\eta_{-}\right)
\end{aligned}
$$

Note that the first nonvanishing term in the low energy expansion is of order $O\left(\omega^{2}\right)$. This holds for all partial waves including the dominant mode $l=0$. Therefore, there is no mode with a nonvanishing low energy greybody factor for the nonminimally coupled scalar. This has a simple explanation: from the equation of motion for the nonminimally coupled scalar, we see that the coupling constant $\xi$ plays a role of an effective mass for the scalar and breaks the infrared enhancement, as mentioned in the introduction.

\section{THE EFFECTS OF VARIOUS PARAMETERS}

There are several parameters in the theory which influence the greybody factor for the nonminimally coupled scalar propagating in the EGB-dS black hole spacetime. These parameters include the non-minimally coupling constant $\xi$, angular momentum number $l$, the spacetime dimension $d$, the cosmological constant $\Lambda$, and the GB coupling constant $\tilde{\alpha}$. In fact, the parameters $\xi, l, d, \Lambda$ have the similar effects on the greybody factor of the EGB-dS black hole as they have for that of the SdS black hole. Therefore, we focus on the effect of the GB coupling constant $\tilde{\alpha}$ on the greybody factor. To analyze their effects more clearly, we plot the dependence of the greybody factor on these parameters and the corresponding effective potentials in the following.

\section{A. The case $\tilde{\alpha}=0$}

For the purpose of comparison, we produce Fig. 1 to show that our results agree with the SdS results (figure 8 in [34]) in the limit $\tilde{\alpha} \rightarrow 0$. From Fig. 1 we see that the suppression of the greybody factor by the angular momentum number $l$ is obvious in the left upper panel, both for minimally or nonminimally coupled scalar. As shown in (3.33), for the dominant mode $l=0$ of the minimally coupled scalar $\xi=0$, we find a nonvanishing greybody factor for the low energy emission. While for the nonminimally coupled scalar, the greybody factor for the low energy mode vanishes. Moreover, $\xi$ decreases the greybody factor when other parameters are fixed. We plot the effective potential in the lower panel to have an intuitive explanation. It can be seen that the effective potential barriers become higher with $\xi$, as a consequence it becomes more difficult for the scalar to transverse the barrier to reach the near horizon region. So the greybody factor decreases with $\xi$.

\section{B. Effects of $\tilde{\boldsymbol{\alpha}}$}

Now we study the effects of the Gauss-Bonnet parameter $\tilde{\alpha}$ on the greybody factor.

\section{Effects of $\tilde{\alpha}$ on different partial modes $l$}

In Fig. 2 we plot the greybody factor for the minimally coupled scalar when $\tilde{\alpha}=0.5$. From the left upper panel, we find the suppression of the greybody factor by the angular momentum number $l$ as well. For the dominant mode $l=0$, there is a nonvanishing greybody factor for the low energy modes. Unlike the case that the greybody factors for zero modes vanish when $\xi \neq 0$, the presence of $\tilde{\alpha}$ makes it have a nonzero value. The greybody factors with respect to $\tilde{\alpha}$ for the dominant mode are shown in the right upper panel. It is obvious that the greybody factor does not vanish when $\omega=0$. Actually, it increases with $\tilde{\alpha}$. We plot the corresponding effective potential in the lower panel to give an intuitive interpretation. The effective potential decreases with $\tilde{\alpha}$ when other parameters are fixed. Thus it becomes easier for the scalar to transverse it and the greybofy factor is enhanced with $\tilde{\alpha}$.

\section{The competition between $\tilde{\alpha}$ and $\xi$}

Since the nonminimally coupling $\xi$ suppresses the Hawking radiation (as we can see in section IVA) while the Gauss-Bonnet term $\tilde{\alpha}$ enhances it, there must be a competition between them. In Fig. 3, we find that when $\xi$ is small ( $\xi=0.1$ in the left panel), $\tilde{\alpha}$ increases the greybody factor. When $\xi$ is large ( $\xi=0.5$ in the right panel), $\tilde{\alpha}$ decreases the greybody factor. This phenomenon appears also for $\xi$ and $\Lambda$ which will be shown in subsection IV B 4 . However, unlike the competition between $\xi$ and $\Lambda$, the competition between $\xi$ and $\tilde{\alpha}$ is too involved for us to have an intuitive analysis from the effective potential.

\section{Effects of $\tilde{\alpha}$ on modes in different dimensional spacetimes $d$}

Now let us study the dependence of the greybody factor on the spacetime dimension $d$ in the presence of $\tilde{\alpha}$. In Fig. 4, we see that the greybody factor is significantly 

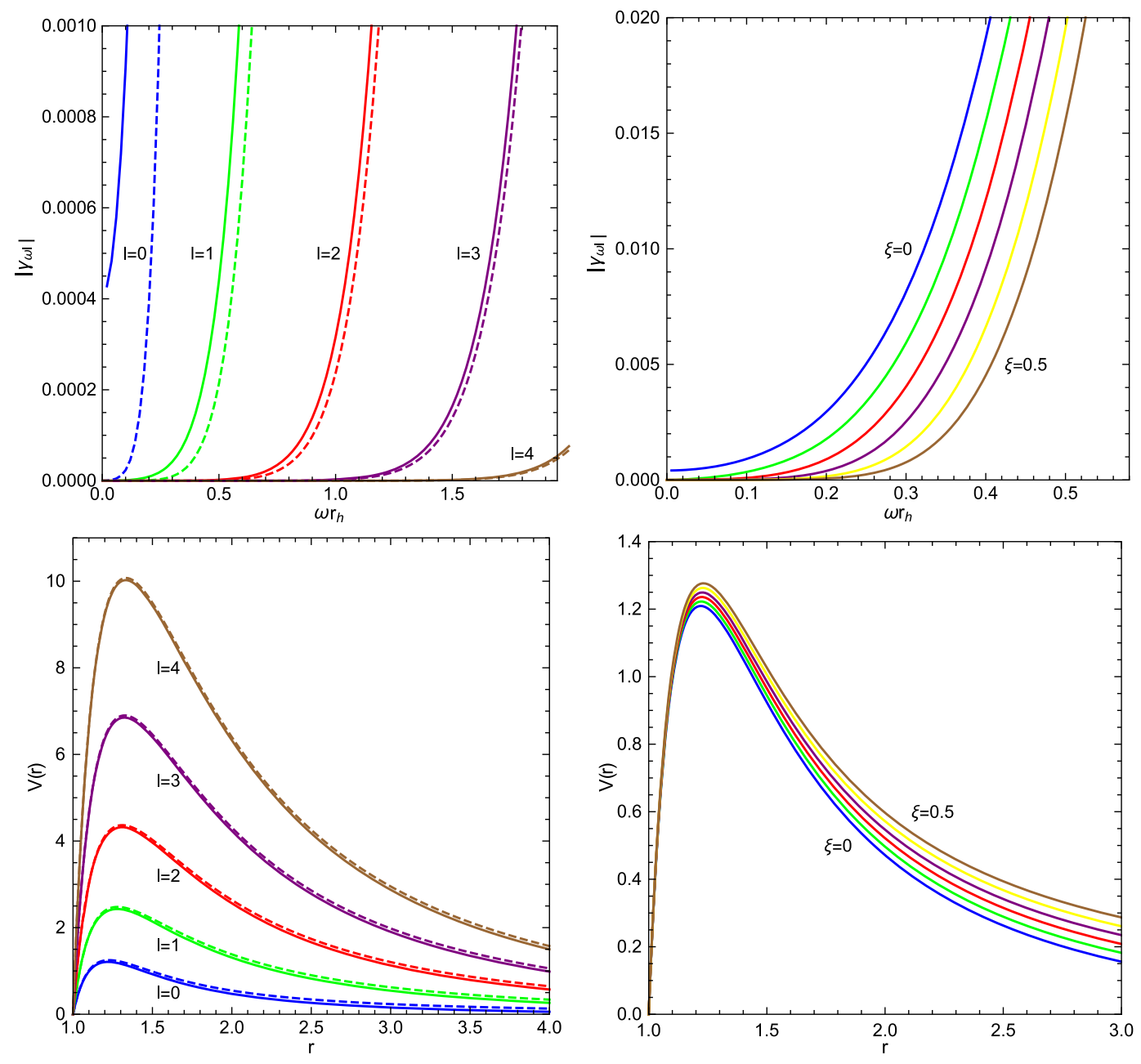

FIG. 1. Effects of parameters $l$ and $\xi$. The greybody factors (upper panel) and corresponding effective potential (lower panel) for the scalar fields when $d=6, \Lambda=0.1, \tilde{\alpha}=0$. Left panel for $l=0,1,2,3,4$ and $\xi=0$ (solid lines) or $\xi=0.3$ (dashed lines). Right panel for $l=0$ and $\xi=0,0.1,0.2,0.3,0.4,0.5$.

suppressed in higher dimensions. For example, for $d=6$, 8,10 the greybody factors for the minimally coupled scalar at $\omega=0$ have values of order $10^{-4}, 10^{-7}$ and $10^{-10}$, respectively. For different dimensions the greybody factor still increases with $\tilde{\alpha}$. We plot the effective potential in the right panel. We see that the potential barrier increases significantly with $d$. Thus it becomes harder for the scalar to transverse the barrier and the greybody factor decreases with $d$. On the other hand, $\tilde{\alpha}$ decreases the potential barrier and so increases the greybody factor. ${ }^{3}$

Note that in Fig. 4 we plot only the greybody factors in the low energy region. In the high energy region the greybody factors decrease to zero which is unreasonable since the high energy modes can transverse the potential barrier easier and the greybody factors should approach to 1 . Thus as we mentioned before, though we do not

\footnotetext{
${ }^{3}$ For the large $d$ behavior of the EGB black holes, one can find the study in $[52,53]$.
}

restrict energy $\omega$ in the derivation of the greybody factors, this matching approach is still limited to low energy region. Moreover, due to the poles of the Gamma functions in the solution, we are not able to obtain the analytical results for odd dimensional spacetimes. A complete analysis is needed and we leave it to future work.

\section{Competition between $\xi$ and $\Lambda$ in the presence of $\tilde{\alpha}$}

We plot the competition between $\xi$ and $\Lambda$ when $\tilde{\alpha}=0.5$ in Fig. 5. As we can see from the left upper panel, when $\xi$ is small, the greybody factor increases with $\Lambda$. However, when $\xi$ is large enough, the greybody factor decreases with $\Lambda$, as shown in the right upper panel. We show the corresponding effective potentials in the lower panels. It is obvious that when $\xi$ is small, the potential barrier decreases with $\Lambda$. The situation is reversed when $\xi$ is large. Thus when $\xi$ is small, $\Lambda$ enhances the greybody factor. When $\xi$ is large enough, $\Lambda$ decreases the greybody factor. The phenomenon is observed similarly in the $\operatorname{SdS}$ 

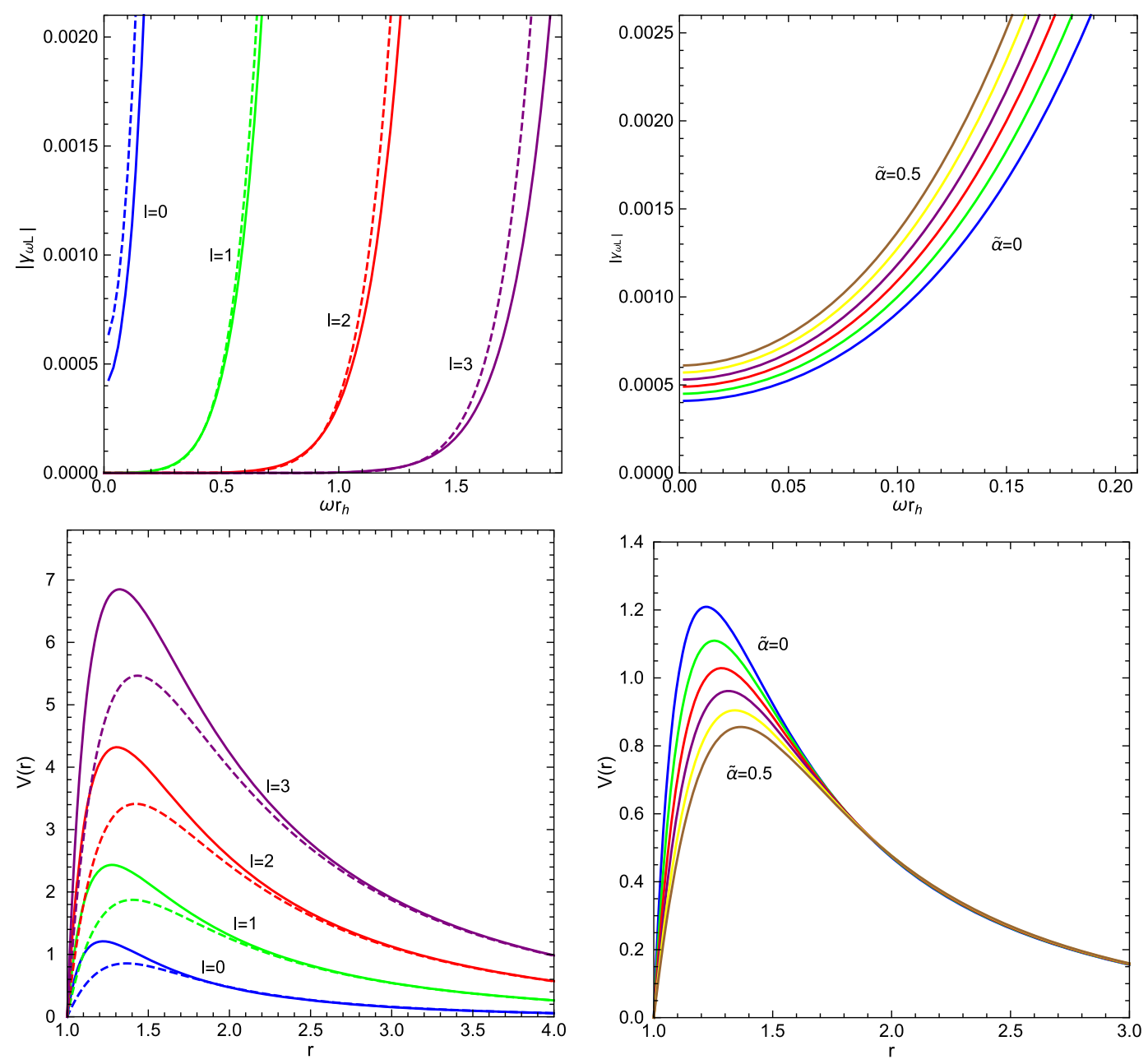

FIG. 2. Effects of $l$ and $\tilde{\alpha}$. The greybody factors (upper panel) and corresponding effective potential (lower panel) for $d=6, \Lambda=0.1$, $\xi=0$. Left panel for $l=0,1,2,3$ and $\tilde{\alpha}=0$ (solid lines) or $\tilde{\alpha}=0.5$ (dashed lines). Right panel for $l=0$ and $\tilde{\alpha}=0$, $0.1,0.2,0.3,0.4,0.5$.
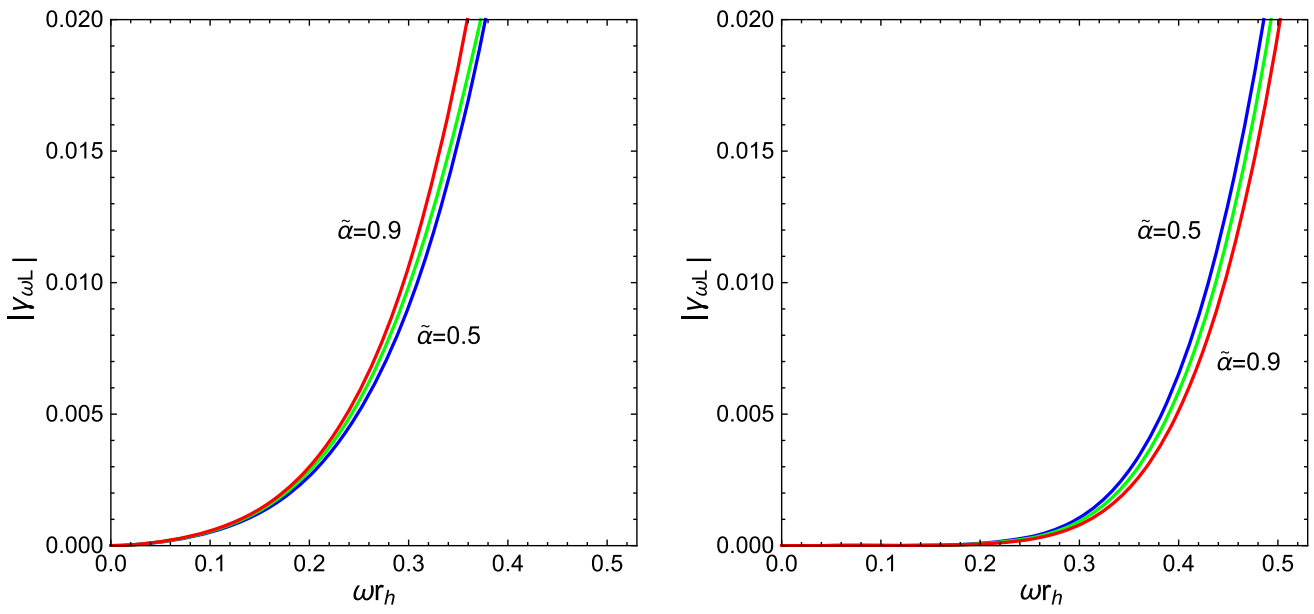

FIG. 3. The competition between $\xi$ and $\tilde{\alpha}$. Greybody factors for $d=6, l=0, \Lambda=0.1, \xi=0.1$ (left) and $\xi=0.5$ (right) with respect to $\tilde{\alpha}=0.5,0.7,0.9$ respectively. 

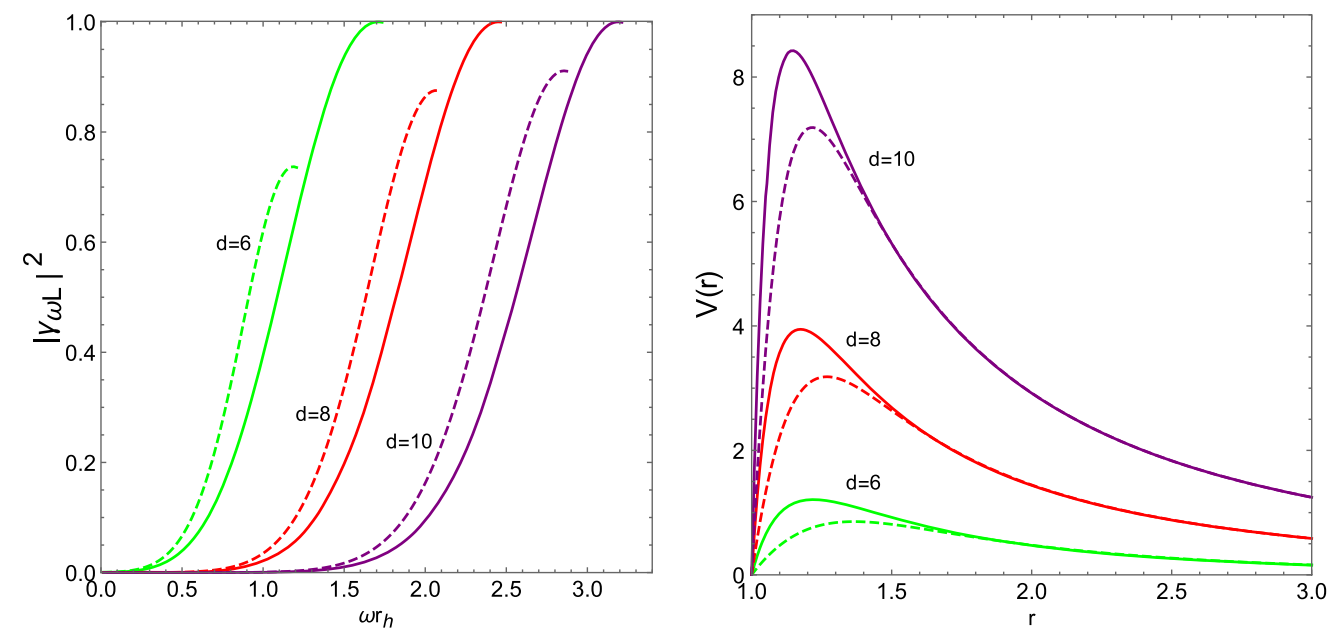

FIG. 4. Effects of $d$ and $\tilde{\alpha}$. The greybody factors (left panel) and corresponding effective potentials (right panel) for $\Lambda=0.1, \xi=0$, $l=0$ and $d=6,8,10$ with $\tilde{\alpha}=0$ (solid lines) or $\tilde{\alpha}=0.5$ (dashed lines).
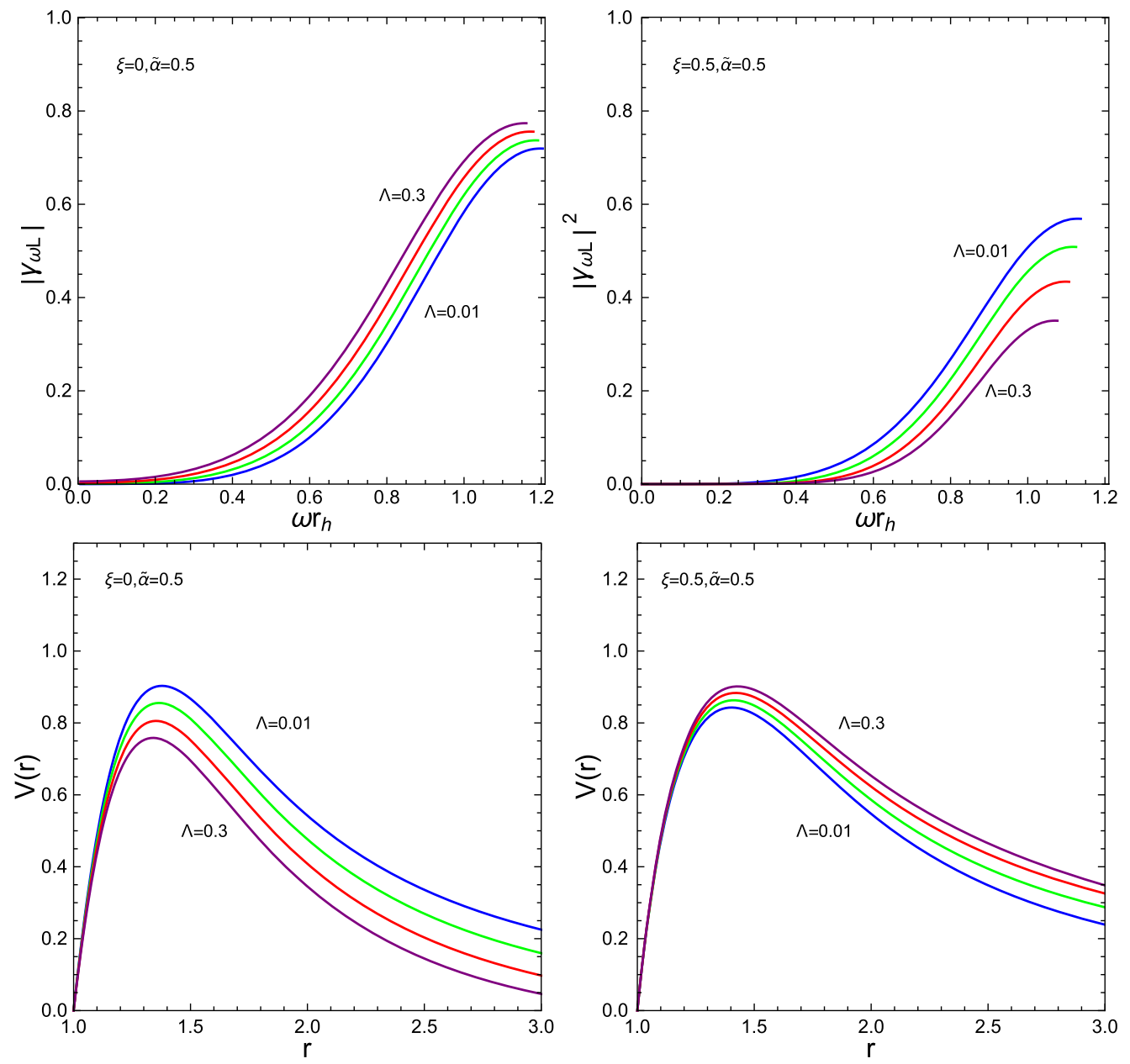

FIG. 5. The competition between $\xi$ and $\Lambda$. The greybody factors (upper panel) and corresponding effective potentials (lower panel) for $d=6, l=0, \tilde{\alpha}=0.5$ with respect to $\Lambda=0.01,0.1,0.2,0.3$. Left panel for $\xi=0$. Right panel for $\xi=0.5$. 
case [34]. It is due to the double roles $\Lambda$ plays in the equations of motion. As a homogeneously energy distributed in the whole spacetime, it subsidizes the energy of emitted particle and hence enhances the radiation. As an effective mass term through the nonminimally coupling term, it suppresses the emission. The competition between these two different contributions leads to the phenomenon we observed.

\section{ENERGY EMISSION RATE OF HAWKING RADIATION}

Greybody factor characterizes the transmissivity of a particular mode. The more direct quantity is the energy emission rate, i.e., the power spectra of Hawking radiation. It is given by $[19,26,54]$

$$
\frac{d^{2} E}{d t d \omega}=\frac{1}{2 \pi} \sum_{l} \frac{N_{l}\left|\gamma_{\omega l}\right|^{2} \omega}{e^{\omega / T_{\mathrm{BH}}}-1}
$$

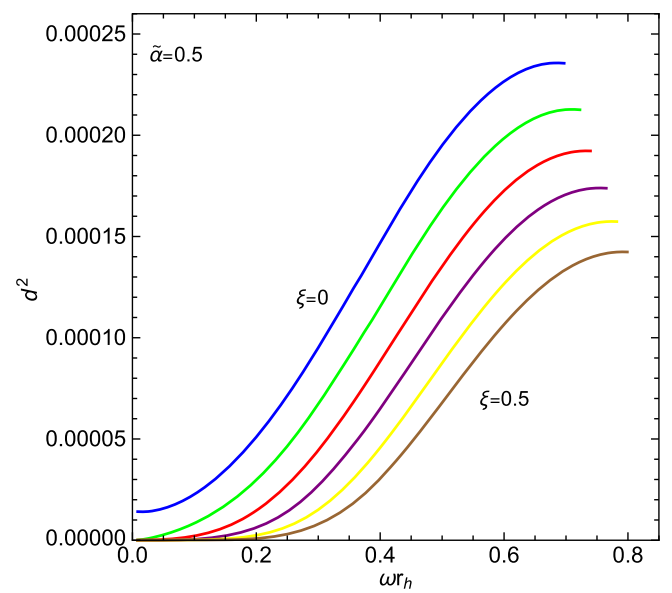

where $\omega$ is the energy of the emitted particle, $\left|\gamma_{\omega l}\right|^{2}$ the greybody factor in Eq. (3.30), $N_{l}=\frac{(2 l+d-3)(l+d-4) !}{l !(d-3) !}$ the multiplicity of states that have the same angular momentum number. $T_{\mathrm{BH}}$ is the normalized temperature of the black hole determined by the surface gravity as $[45,55]$

$$
T_{\mathrm{BH}}=\frac{1}{\sqrt{h\left(r_{0}\right)}} \frac{1}{4 \pi}\left[\frac{(d-2)\left[(d-3) r_{h}^{2}+(d-5) \tilde{\alpha}\right]-2 \Lambda r_{h}^{4}}{(d-2) r_{h}\left(r_{h}^{2}+2 \tilde{\alpha}\right)}\right] .
$$

Here $r_{0}$ is the position where $h(r)$ is extreme. We mainly consider the effects of $\xi$ and $\alpha$ on the power spectra in this section. Since modes higher than $l>6$ have contributions many orders of magnitude lower than those of the $l \leq 6$ modes, their contributions to the energy emission rate are ignored safely.

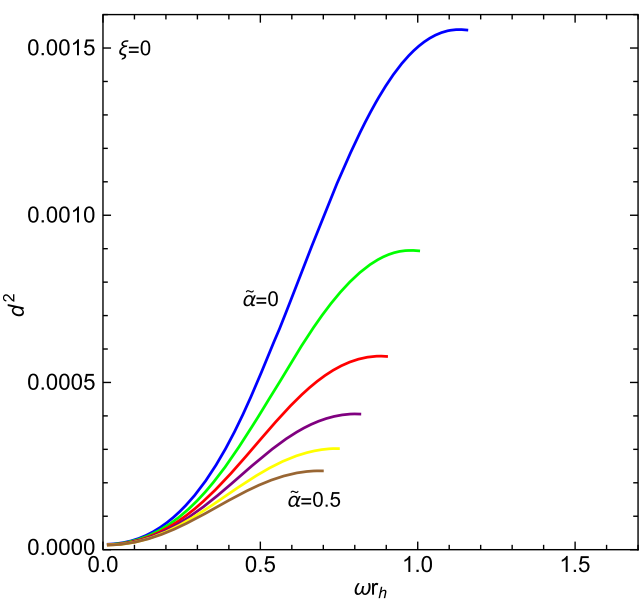

FIG. 6. Power spectra of Hawking radiation for $d=6, \tilde{\alpha}=0.5, \Lambda=0.1$ with respect to $\tilde{\alpha}=0.5, \xi=0,0.1,0.2,0.3,0.4,0.5$ (left panel) and $\xi=0$ and $\tilde{\alpha}=0,0.1,0.2,0.3,0.4,0.5$ (right panel).
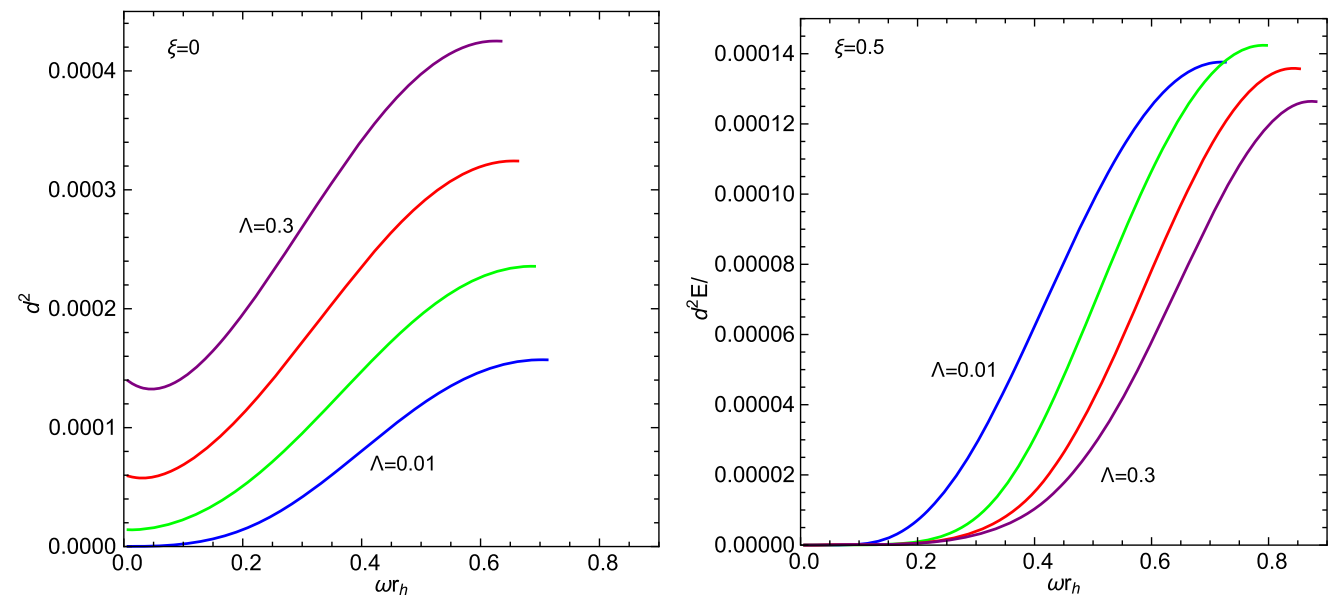

FIG. 7. Power spectra of Hawking radiation for $d=6, \tilde{\alpha}=0.5$ with respect to $\Lambda=0.01,0.1,0.2,0.3$. Left panel for $\xi=0$. Right panel for $\xi=0.5$. 


\section{A. The effects of $\xi$ and $\alpha$}

We plot the dependence of power spectra on $\xi$ and $\alpha$ in Fig. 6.

It has been found that $\xi$ suppresses the Hawking radiation in SdS background. In the left panel, we see that $\xi$ still suppresses the Hawking radiation in EGB-dS background. This behavior is coincident with that of greybody factor in Fig. 1. Since the greybody factor decreases with $\xi$, as can be seen from Eq. (5.1), the power spectra decreases when other parameters are fixed.

In the right panel, we see that $\tilde{\alpha}$ also suppresses the Hawking radiation. Since $\tilde{\alpha}$ increases the greybody factor in Fig. 2, it seems strange at first sight. However, the power spectra also depends on the temperature of the black hole. It can be proved easily that the normalized temperature in Eq. (5.2) decreases with $\tilde{\alpha}$. This leads to the decrease of the power spectra with $\tilde{\alpha}$ finally.

\section{B. The competition between $\xi$ and $\Lambda$}

We have observed that there is a competition between the contribution of $\xi$ and $\Lambda$ for greybody factor in subsection IV B 4. In fact, they have the similar competition for power spectra of Hawking radiation. We plot their influences on the power spectra in Fig. 7. It is obvious that when $\xi$ is small, $\Lambda$ increases the Hawking radiation. When $\xi$ is large enough, $\Lambda$ decreases the Hawking radiation. Note that the existence of EGB coupling constant does not change this behavior qualitatively.

\section{CONCLUSION AND DISCUSSION}

We studied the greybody factors of the Hawking radiation for the minimally and nonminimally coupled scalar fields in a higher dimensional Einstein-Gauss-Bonnet-dS black hole spacetime. Solving the equations of motion near the event horizon and cosmological horizon separately and matching them in the intermediate region, we derived an analytical formula for the greybody factors when the cosmological constant is small. The larger the distance between the cosmological horizon and the event horizon, the more accurate the analytical formula.

The effects of various parameters, such as the angular momentum number $l$, the nonminimally coupling constant $\xi$, the cosmological constant $\Lambda$, the GB coupling constant $\tilde{\alpha}$, and the spacetime dimension $d$, on the greybody factor were studied in detail. We found that when other parameters are fixed, similar to the case without the GB term, $l, \xi$ or $d$ suppresses the greybody factor separately. However, the GB coupling constant $\tilde{\alpha}$ enhances the greybody factor. We analyzed the competition between $\xi$ and $\tilde{\alpha}$. We also studied their effects on the power spectra of Hawking radiation, and found that both of them suppressed the power spectra. The effect of the cosmological constant $\Lambda$ is more involved. When $\xi$ is small, it enhances the greybody factor. When $\xi$ is large enough, it suppresses the greybody factor. We plotted the effective potentials to give some intuitive explanations to the phenomenons we observed.

For the dominant mode $l=0$, the greybody factor for the minimally coupled scalar is nonvanishing when $\omega=0$. This feature is characteristic for the free massless scalar propagating in the dS black hole spacetime. For the EGB-dS black hole, the presence of GB constant $\tilde{\alpha}$ preserves this feature qualitatively. But quantitatively, it increases the greybody factor at $\omega=0$.

For the nonminimally coupled scalar, the greybody factors are of order $O\left(\omega^{2}\right)$ and vanish for the low energy modes for all the partial modes $l$ including $l=0$. This can be explained by the fact that for the non-minimally coupled scalar, $\xi$ plays the role of effective mass and hinders the Hawking radiation when $\omega \rightarrow 0$. We obtained the coefficient of the term at $O\left(\omega^{2}\right)$ for the EGB-dS black hole background.

As we mentioned in the context, the results we obtained is only be valid in the low energy region, by using the numerical method we may be able to obtain the greybody in the high energy region. We leave this work to future.

\section{ACKNOWLEDGMENTS}

We are appreciated Nikalaos Pappas and Panagiota Kanti for their correspondences. C. Y. Zhang is supported by National Postdoctoral Program for Innovative Talents BX201600005. B.Chen and P. C. Li were in part supported by NSFC Grant No. 11275010, No. 11325522 and No. 11735001.
[1] J. M. Bardeen, B. Carter, and S. W. Hawking, The four laws of black hole mechanics, Commun. Math. Phys. 31, 161 (1973).

[2] S. W. Hawking, Particle creation by black holes, Commun. Math. Phys. 43, 199 (1975).

[3] S. W. Hawking, Black holes and thermodynamics, Phys. Rev. D 13, 191 (1976).
[4] P. C. Argyres, S. Dimopoulos, and J. March-Russell, Black holes and submillimeter dimensions, Phys. Lett. B 441, 96 (1998).

[5] N. Arkani-Hamed, S. Dimopoulos, and G. R. Dvali, The hierarchy problem and new dimensions at a millimeter, Phys. Lett. B 429, 263 (1998). 
[6] I. Antoniadis, N. Arkani-Hamed, S. Dimopoulos, and G. R. Dvali, New dimensions at a millimeter to a Fermi and superstrings at a TeV, Phys. Lett. B 436, 257 (1998).

[7] L. Randall and R. Sundrum, A Large Mass Hierarchy from a Small Extra Dimension, Phys. Rev. Lett. 83, 3370 (1999).

[8] L. Randall and R. Sundrum, An Alternative to Compactification, Phys. Rev. Lett. 83, 4690 (1999).

[9] S. B. Giddings and S. D. Thomas, High-energy colliders as black hole factories: The End of short distance physics, Phys. Rev. D 65, 056010 (2002).

[10] S. Dimopoulos and G. L. Landsberg, Black Holes at the LHC, Phys. Rev. Lett. 87, 161602 (2001).

[11] G. L. Landsberg, Black holes at future colliders and beyond, J. Phys. G 32, R337 (2006).

[12] D.-C. Dai, G. Starkman, D. Stojkovic, C. Issever, E. Rizvi, and J. Tseng, BlackMax: A black-hole event generator with rotation, recoil, split branes, and brane tension, Phys. Rev. D 77, 076007 (2008).

[13] P. Kanti, Black holes at the LHC, Lect. Notes Phys. 769, 387 (2009).

[14] J. L. Feng and A. D. Shapere, Black Hole Production by Cosmic Rays, Phys. Rev. Lett. 88, 021303 (2001).

[15] R. Emparan, M. Masip, and R. Rattazzi, Cosmic rays as probes of large extra dimensions and TeV gravity, Phys. Rev. D 65, 064023 (2002).

[16] A. Ringwald and $\mathrm{H}$. Tu, Collider versus cosmic ray sensitivity to black hole production, Phys. Lett. B 525, 135 (2002).

[17] L. A. Anchordoqui, J. L. Feng, H. Goldberg, and A. D. Shapere, Black holes from cosmic rays: Probes of extra dimensions and new limits on TeV-scale gravity, Phys. Rev. D 65, 124027 (2002).

[18] G. L. Landsberg, Black holes at future colliders and in cosmic rays, Eur. Phys. J. C 33, S927 (2004).

[19] P. Kanti, Black holes in theories with large extra dimensions: A review, Int. J. Mod. Phys. A 19, 4899 (2004).

[20] T. Harmark, J. Natario, and R. Schiappa, Greybody factors for d-dimensional black holes, Adv. Theor. Math. Phys. 14, 727 (2010).

[21] P. Kanti and E. Winstanley, Hawking radiation from higherdimensional black holes, Quantum Aspects of Black Holes 178, 229 (2015).

[22] D. N. Page, Particle emission rates from a black hole: Massless particles from an uncharged, nonrotating hole, Phys. Rev. D 13, 198 (1976).

[23] S. R. Das, G. Gibbons, and S. D. Mathur, Universailty of Low Energy Absorption Cross-sections for Black Holes, Phys. Rev. Lett. 78, 417 (1997).

[24] A. Higuchi, Low-frequency scalar absorption cross sections for stationary black holes, Classical Quantum Gravity 18, L139 (2001); Addendum, Classical Quantum Gravity 19, 599 (2002).

[25] S. Chen and J. Jing, Greybody factor for a scalar field coupling to Einstein's tensor, Phys. Lett. B 691, 254 (2010).

[26] P. Kanti, J. Grain, and A. Barrau, Bulk and brane decay of a $(4+n)$-dimensional Schwarzschild-De-Sitter black hole: Scalar radiation, Phys. Rev. D 71, 104002 (2005).

[27] P. R. Brady, C. M. Chambers, W. Krivan, and P. Laguna, Telling tails in the presence of a cosmological constant, Phys. Rev. D 55, 7538 (1997).
[28] L. C. B. Crispino, A. Higuchi, E. S. Oliveira, and J. V. Rocha, Greybody factors for non-minimally coupled scalar fields in Schwarzschild-de Sitter spacetime, Phys. Rev. D 87, 104034 (2013).

[29] D. Lovelock, The Einstein tensor and its generalizations, J. Math. Phys. (N.Y.) 12, 498 (1971).

[30] D. G. Boulware and S. Deser, String-Generated Gravity Models, Phys. Rev. Lett. 55, 2656 (1985).

[31] A. Barrau, J. Grain, and S. O. Alexeyev, Gauss-Bonnet black holes at the LHC: Beyond the dimensionality of space, Phys. Lett. B 584, 114 (2004).

[32] J. Grain, A. Barrau, and P. Kanti, Exact results for evaporating black holes in curvature-squared lovelock gravity: Gauss-Bonnet greybody factors, Phys. Rev. D 72, 104016 (2005).

[33] R. A. Konoplya and A. Zhidenko, Long life of GaussBonnet corrected black holes, Phys. Rev. D 82, 084003 (2010).

[34] P. Kanti, T. Pappas, and N. Pappas, Greybody factors for scalar fields emitted by a higher-dimensional Schwarzschildde-Sitter black-hole, Phys. Rev. D 90, 124077 (2014).

[35] T. Pappas, P. Kanti, and N. Pappas, Hawking radiation spectra for scalar fields by a higher-dimensional Schwarzschildde-Sitter black hole, Phys. Rev. D 94, 024035 (2016).

[36] P. Gonzalez, E. Papantonopoulos, and J. Saavedra, ChernSimons black holes: scalar perturbations, mass and area spectrum and greybody factors, J. High Energy Phys. 08 (2010) 050.

[37] P. A. Gonzalez and J. Saavedra, Comments on absorption cross section for Chern-Simons black holes in five dimensions, Int. J. Mod. Phys. A 26, 3997 (2011).

[38] R. Jorge, E. S. de Oliveira, and J. V. Rocha, Greybody factors for rotating black holes in higher dimensions, Classical Quantum Gravity 32, 065008 (2015).

[39] R. Dong and D. Stojkovic, Greybody factors for a black hole in massive gravity, Phys. Rev. D 92, 084045 (2015).

[40] C. A. Sporea and A. Borowiec, Low energy Greybody factors for fermions emitted by a Schwarzschild-de Sitter black hole, Int. J. Mod. Phys. D 25, 1650043 (2016).

[41] I. Sakalli and O. A. Aslan, Absorption cross-section and decay rate of rotating linear dilaton black holes, Astropart. Phys. 74, 73 (2016).

[42] J. Ahmed and K. Saifullah, Greybody factor of scalar field from Reissner-Nordstrom-de Sitter black hole, arXiv: 1610.06104.

[43] G. Panotopoulos and A. Rincon, Greybody factors for a nonminimally coupled scalar field in BTZ black hole background, Phys. Lett. B 772, 523 (2017).

[44] Y.-G. Miao and Z.-M. Xu, Hawking radiation of fivedimensional charged black holes with scalar fields, Phys. Lett. B 772, 542 (2017).

[45] P. Kanti and T. Pappas, Effective temperatures and radiation spectra for a higher-dimensional Schwarzschild-de Sitter black hole, Phys. Rev. D 96, 024038 (2017).

[46] G. Panotopoulos and Á. Rincón, Greybody factors for a minimally coupled massless scalar field in Einstein-BornInfeld dilaton spacetime, Phys. Rev. D 96, 025009 (2017).

[47] T. Pappas and P. Kanti, Schwarzschild-de Sitter spacetime: The role of temperature in the emission of Hawking radiation, Phys. Lett. B 775, 140 (2017). 
[48] G. Panotopoulos and A. Rincon, Quasinormal modes of black holes in Einstein-power-Maxwell theory, arXiv:1711.04146 [Phys. Rev. D (to be published)].

[49] R. A. Konoplya and A. Zhidenko, (In)stability of Ddimensional black holes in Gauss-Bonnet theory, Phys. Rev. D 77, 104004 (2008).

[50] M. A. Cuyubamba, R. A. Konoplya, and A. Zhidenko, Quasinormal modes and a new instability of EinsteinGauss-Bonnet black holes in the de Sitter world, Phys. Rev. D 93, 104053 (2016).

[51] R. A. Konoplya and A. Zhidenko, The portrait of eikonal instability in Lovelock theories, J. Cosmol. Astropart. Phys. 05 (2017) 050.
[52] B. Chen, Z.-Y. Fan, P. Li, and W. Ye, Quasinormal modes of Gauss-Bonnet black holes at large D, J. High Energy Phys. 01 (2016) 085.

[53] B. Chen and P.-C. Li, Static Gauss-Bonnet black holes at large D, J. High Energy Phys. 05 (2017) 025.

[54] C. M. Harris and P. Kanti, Hawking radiation from a $(4+n)$-dimensional black hole: Exact results for the Schwarzschild phase, J. High Energy Phys. 10 (2003) 014.

[55] R. Bousso and S. W. Hawking, Pair creation of black holes during inflation, Phys. Rev. D 54, 6312 (1996). 